\title{
Estimating the cost of illness in colorectal cancer patients who were hospitalized for severe chemotherapy-induced diarrhea
}

\author{
George Dranitsaris MPharm FCSHP ${ }^{1}$, Jean Maroun MD², Amil Shah MD ${ }^{3}$
}

\begin{abstract}
G Dranitsaris, J Maroun, A Shah. Estimating the cost of illness in colorectal cancer patients who were hospitalized for severe chemotherapy-induced diarrhea. Can J Gastroenterol 2005;19(2):83-87.
\end{abstract}

BACKGROUND: Previous studies have suggested that grade III/IV diarrhea is a common complication in colorectal cancer, occurring in $20 \%$ to $30 \%$ of patients receiving chemotherapy. In some of these patients, hospitalization for supportive care is often required. However, the impact that these hospitalized patients have on overall use of health care resources has not been quantified. In the present study, a cost of illness analysis was conducted to estimate the overall cost of patients with colorectal cancer who were hospitalized for supportive care secondary to severe diarrhea.

METHODS: This was a retrospective cohort study consisting of patients with colorectal cancer that had received fluoropyrimidines, irinotecan or oxaliplatin (or a combination thereof) and had developed grade III or IV diarrhea that resulted in hospital admission for supportive care. Data collection included patient demographics, disease-related information and use of health care resources to manage the grade III/IV diarrhea event.

RESULTS: Patients had a mean age of 64.2 years, and 32 of 63 (50.8\%) were receiving adjuvant chemotherapy with a curative intent. The severe diarrhea developed after the first cycle of chemotherapy in $58 \%$ of the patients and contributed to a dose reduction, change or discontinuation of chemotherapy in $9.5 \%$, $15.9 \%$ and $34.2 \%$ of patients, respectively. Overall, the median length of hospital stay was eight days (range one to 49 days) translating to a mean cost of $\$ 8,230$ per patient ( $95 \%$ CI $\$ 6,519$ to $\$ 9,942$ ). The diarrhea successfully resolved in 54 of 63 patients $(85.7 \%)$.

CONCLUSIONS: Severe diarrhea requiring hospital admission is a costly and potentially fatal complication of chemotherapy in colorectal cancer. The identification of predictive factors and the implementation of prophylactic measures could reduce the morbidity and mortality associated with diarrhea.

Key Words: Cancer; Chemotherapy; Cost; Diarrhea

\section{Estimation du coût de la maladie chez les patients atteints d'un cancer colorectal hospi- talisés en raison d'une grave diarrhée provo- quée par la chimiothérapie}

HISTORIQUE : D'après des études antérieures, la diarrhée de grade III ou IV est une complication courante du cancer colorectal, qui se manifeste chez $20 \%$ à $30 \%$ des patients sous chimiothérapie. Chez certains de ces patients, une hospitalisation s'impose souvent pour administrer des soins de soutien. Cependant, les effets de ces patients hospitalisés sur l'utilisation globale des ressources de santé n'ont pas été quantifiés. Dans la présente étude, une analyse du coût de la maladie a été effectuée pour estimer le coût global des patients atteints de cancer colorectal hospitalisés afin de recevoir des soins de soutien par suite d'une grave diarrhée. MÉTHODOLOGIE : Il s'agissait d'une étude rétrospective de cohorte composée de patients atteints de cancer colorectal qui avaient pris des fluoropyrimidines, de l'irinotécan ou de l'oxaliplatin (ou une association de ces médicaments) et avaient développé une diarrhée de groupe III ou IV justifiant une hospitalisation afin de recevoir des soins de soutien. La collecte de données incluait la démographie du patient, l'information reliée à la maladie et l'utilisation des ressources de santé pour prendre en charge la diarrhée de grade III ou IV.

RÉSULTATS : Les patients avaient un âge moyen de 64,2 ans, et 32 des $63(50,8 \%)$ prenaient une chimiothérapie adjuvante dans un but curatif. La diarrhée grave est apparue après le premier cycle de chimiothérapie chez $58 \%$ des patients et a suscité une diminution de la dose, une modification ou un arrêt de la chimiothérapie chez 9,5\%,15,9\% et 34,2\% des patients, respectivement. Dans l'ensemble, la durée médiane d'hospitalisation était de huit jours (fourchette de un à 49 jours), se traduisant par un coût moyen de 8230 \$ par patient (95\% IC 6519 \$ à 9942 \$). Cinquante-quatre des 63 patients $(85,7 \%)$ ont bénéficié d'une résolution de la diarrhée.

CONCLUSIONS : Une diarrhée grave exigeant une hospitalisation est une complication coûteuse, au potentiel fatal, de la chimiothérapie chez les patients atteints de cancer colorectal. Le dépistage des facteurs prédicteurs et l'adoption de mesures prophylactiques pourraient réduire la morbidité et la mortalité associées à la diarrhée.
$\mathrm{T}$ he populations of North America and Europe are aging. This is of concern to health care authorities because cancer is a disease that usually occurs later in life. It has been estimated that in Canada, $60 \%$ of all new cancers occur in people over the age of 65 years (1). Because the population is aging, a sharp increase in the number of new cases during the next
10 to 20 years is to be expected. Cancer is also considered to be a chronic disease where some patients can live for five to 10 years after the initial diagnosis. Furthermore, the intensity of chemotherapy in both the adjuvant and palliative setting has also increased over the past decade, resulting in elevated toxicity. From the perspective of a cancer treatment centre,

${ }^{1}$ Cancer Care Ontario, Toronto; ${ }^{2}$ Ottawa Regional Cancer Centre, Ottawa, Ontario; ${ }^{3}$ British Columbia Cancer Centre, Vancouver,

British Columbia

Correspondence: George Dranitaris, Consultant pharmacist, Cancer Care Ontario, 429 Danforth Avenue, Suite 476, Toronto, Ontario

M4K 1P1. Telephone 416-461-2720, fax 416-461-4735, e-mail gdranit@ca.inter.net

Received for publication June 16, 2004. Accepted October 22,2004 
these factors will contribute to greater demands on health care staff to provide quality service and on the overall health care budget. To adequately meet these increased needs, new interventions need to be identified that will allow chemotherapy to be delivered in an efficient manner while minimizing debilitating toxicity.

Of the many side effects of chemotherapy, diarrhea can be debilitating and potentially fatal $(2,3)$. If poorly controlled, diarrhea can contribute to chemotherapy dosage delays and reductions, costly hospitalizations and even patient death $(3,4)$. The ability to adequately control or even prevent the onset of severe chemotherapy-induced diarrhea (CID) with agents such as octreotide in the outpatient setting may allow the optimization of chemotherapy dose intensity and prevent costly hospitalizations (5). The latter phenomenon is particularly important to drug formulary committees, who must consider both the costs and benefits of interventions such as octreotide.

To provide some of this important information to formulary committees, the current study was designed to measure the overall cost of hospitalization secondary to severe uncontrolled diarrhea. The population selected for analysis consisted of patients with colorectal cancer receiving chemotherapy in the adjuvant or advanced disease setting. This population was selected because up to $50 \%$ of such patients can develop diarrhea by the end of their chemotherapy (4). Therefore, the ability to prevent or at least lessen the intensity of severe diarrhea in these patients could have a profound impact on the overall cost of care. In the present study, a cost of illness analysis was conducted to estimate the cost impact of severe diarrhea requiring hospitalization in colorectal cancer patients receiving adjuvant or palliative chemotherapy.

\section{METHODS}

\section{Study design}

This was a two-centre, retrospective cohort study consisting of patients with colorectal cancer who received chemotherapy in the adjuvant setting or for metastatic disease. To be eligible, patients had to be adults with a diagnosis of colorectal cancer and had received fluoropyrimidines, irinotecan or oxaliplatin (or a combination thereof). To quantify the overall cost impact of severe CID, patients must have been hospitalized following an episode of grade III or IV diarrhea according to the National Cancer Institute Common Toxicity Criteria (NCI-CTC) (6). NCI-CTC grade III diarrhea is defined as seven to nine stools/day, incontinence or severe cramping. Diarrhea is classified as grade IV if there is at least 10 stools/day, a need for parenteral support for dehydration or if grossly bloody diarrhea develops. Patients who developed only grade I or II diarrhea according to NCI-CTC were not included in the study.

\section{Data collection}

Patient charts were reviewed for eligibility and selected consecutively. For patients meeting the inclusion criteria, clinical and resource utilization information was collected from the charts using a standardized data collection instrument. The data collection included patient demographic and disease-related information, previous and current chemotherapy, and NCI-CTC grade of diarrhea including the number of stools, time of onset, duration and all other symptoms associated with the diarrhea episode (eg, severe cramping, grossly bloody diarrhea, etc). In addition to the clinical information, use of health care resources to manage the grade III/IV CID event was also collected. This consisted of unscheduled visits to the oncology clinic or emergency departments, hospitalization for supportive care, all medical interventions to manage the diarrhea (eg, octreotide, antibiotics), laboratory and microbiological tests, diagnostic imaging procedures, nursing time, number of physician visits in hospitalized patients and number of specialist consultations that were directly related to the diarrhea episode. All patient outcomes (eg, successful resolution of diarrhea, death, etc) were also recorded as well as the impact of the diarrhea event on subsequent chemotherapy.

Resource use items were then converted into costs to estimate the mean cost per episode of severe CID. Unit costs for drugs, materials and other related hospital resources (eg, laboratory and diagnostic tests) were obtained in 2004 from the Toronto Sunnybrook Regional Cancer Centre, and from the University Health Network in Toronto, Ontario. The cost of daily hospitalization for a Canadian oncology centre (\$644/day) was obtained from Doyle et al (7). Physician's fees for services were obtained from the Schedule of Benefits: Physician Services under the Health Insurance Act, Ontario Ministry of Health, 1998. All costs in the current analysis were reported in Canadian dollars (\$CDN1=\$US0.79 or EUR0.65 as of January 2004).

\section{Sample size and data analysis}

The study was designed to determine overall resource use and total cost of grade III/IV CID requiring hospital admission under a socialized health care system. To meet the primary objective, the final sample size for the present cohort study was 63 patients. With such a sample size, the overall cost of CID was measured with a precision that extended to $\pm \$ 900$, with a $95 \%$ probability. All patient and clinical data are presented descriptively as means, medians or proportions. All of the statistical analyses were performed using Stata, release 7.0 (Stata Corp, USA).

One of the challenges in estimating the overall cost of severe CID requiring hospital admission is that other side effects of chemotherapy (eg, stomatitis, febrile neutropenia and uncontrolled emesis) often occur alongside severe diarrhea. This was especially apparent with febrile neutropenia, in which patients are often hospitalized for empirical antibiotic therapy. To avoid this potential bias, only resource items that were directly related to managing the diarrhea episode were considered. Empirical broad spectrum antibiotics for neutropenic fever and total parenteral nutrition secondary to severe stomatitis were not included. A sensitivity analysis was also conducted to further limit this potential bias. Specifically, the data were reanalyzed after patients with concomitant neutropenia, febrile neutropenia, stomatitis and severe uncontrolled emesis were systematically removed.

\section{RESULTS}

Patients who met the eligibility criteria had a mean age of 64.2 years and $50.8 \%$ were male. The most prominent sites of cancer were the cecum, sigmoid colon and rectum, with $81.4 \%$ of tumours being well- or moderately differentiated (Table 1). Over $98 \%$ of patients had previously received surgery, but only $39.4 \%$ had subsequently received chemotherapy. At the time of the CID event, approximately $44.5 \%$ of patients were receiving 5-fluorouracil as a continuous infusion or as bolus injections with leucovorin, and single agent irinotecan was administered in $12.7 \%$ of cases. The remaining patients were receiving either single agent capecitabine, or combinations of capecitabine, irinotecan, oxaliplatin and raltitrexed. In addition, $11.5 \%$ of patients were receiving concurrent radiotherapy. 
TABLE 1

Demographic and clinical characteristics of patients with grade III/IV chemotherapy-induced diarrhea

\begin{tabular}{|c|c|}
\hline Parameter & Distribution $(n=63)$ \\
\hline Mean age, years (range) & $64.2(36$ to 100$)$ \\
\hline Male, \% & 50.8 \\
\hline \multicolumn{2}{|l|}{ Primary neoplasm, \% } \\
\hline Cecum & 19.4 \\
\hline Sigmoid colon & 24.2 \\
\hline Transverse colon & 6.4 \\
\hline Ascending colon & 8.1 \\
\hline Rectosigmoid junction & 9.7 \\
\hline Rectum & 22.6 \\
\hline Other & 9.6 \\
\hline \multicolumn{2}{|l|}{ Tumour grade, $\%$} \\
\hline Well-differentiated & 11.9 \\
\hline Moderately differentiated & 69.5 \\
\hline Poorly differentiated & 15.2 \\
\hline Unknown & 3.4 \\
\hline \multicolumn{2}{|l|}{ Previous treatment, $\%$} \\
\hline None & 1.6 \\
\hline Surgery & 59.0 \\
\hline Surgery + chemotherapy & 24.6 \\
\hline Surgery + chemotherapy + radiation & 14.8 \\
\hline \multicolumn{2}{|l|}{ Current chemotherapy, \% } \\
\hline 5 -fluorouracil by continuous infusion & 6.4 \\
\hline 5-fluorouracil + leucovorin & 38.1 \\
\hline Irinotecan & 12.7 \\
\hline Other* & 42.8 \\
\hline Concurrent radiotherapy, \% & 11.5 \\
\hline \multicolumn{2}{|l|}{ Intent of current chemotherapy, \% } \\
\hline Adjuvant & 50.8 \\
\hline Palliative & 49.2 \\
\hline
\end{tabular}

${ }^{*}$ Capecitabine, raltitrexed, 5-fluorouracil + irinotecan, 5-fluorouracil + oxaliplatin, etc

Overall, the intent of chemotherapy was curative in $50.8 \%$ of patients (Table 1).

The severe diarrhea developed after the first cycle of chemotherapy in $58 \%$ of patients (Figure 1). When the CID event was documented, patients had a mean absolute neutrophil count of $2.5 \times 10^{6} / \mathrm{L}$ and hemoglobin of $112 \mathrm{~g} / \mathrm{L}$. The prevalence of grade IV diarrhea was approximately threefold higher than grade III and the median time to the CID event was five days following the completion of chemotherapy (Table 2). Approximately $33.3 \%$ of patients had seven to nine stools on the worst day of diarrhea and $22.2 \%$ of patients had more than 15 stools per day. The diarrhea was of variable duration but it lasted more than seven days in $45.8 \%$ of patients. The diarrhea event was also associated with other symptoms such as cramping $(39.7 \%)$, bloody stools $(9.5 \%)$, uncontrolled emesis $(20.6 \%)$, fever requiring antibiotic support $(30.2 \%)$, stomatitis $(7.9 \%)$, neutropenia $(59.7 \%)$ and febrile neutropenia $(39.7 \%)$. Before their hospitalization for uncontrolled diarrhea, $58.7 \%$ of patients had an unscheduled return to the oncology clinic and $42.9 \%$ had to visit their nearest hospital emergency department for symptom control.

Most patients (37 of 63) received loperamide and diphenoxylate either alone or in combination for the management

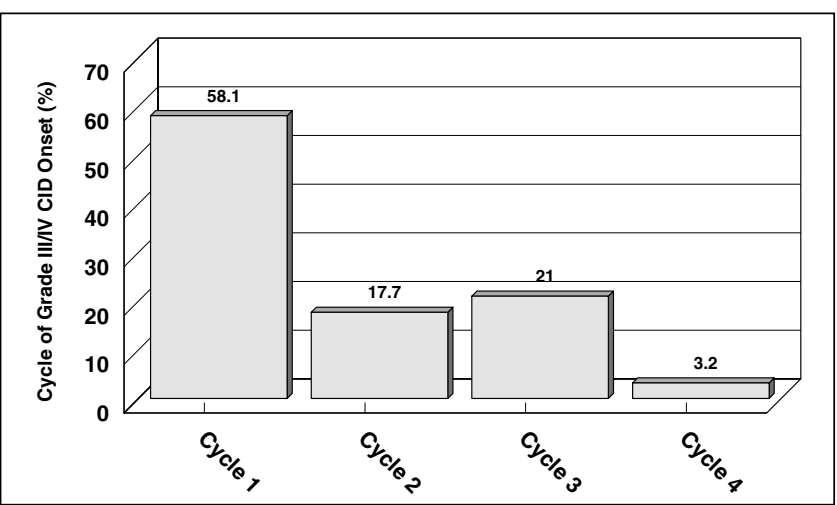

Figure 1) Cycle of grade III/IV chemotherapy-induced diarrhea (CID) onset

TABLE 2

Characteristics of the grade III/IV chemotherapy-induced diarrhea (CID) event and associated conditions

\begin{tabular}{|c|c|}
\hline Parameter & Distribution $(n=63)$ \\
\hline Mean absolute neutrophil count, $\times 10^{6} / \mathrm{L}$ (range) & $2.5(0.1$ to 16.9$)$ \\
\hline Mean hemoglobin, g/L (range) & $112(73$ to 186$)$ \\
\hline Grade III CID, \% & 23.8 \\
\hline Grade IV CID, \% & 76.2 \\
\hline $\begin{array}{l}\text { Median time to diarrhea from end } \\
\text { of chemotherapy cycle, days (range) }\end{array}$ & $5(0$ to 31$)$ \\
\hline \multicolumn{2}{|l|}{ Total number of stools on the worst day, \% } \\
\hline 7 to 9 stools per day & 33.3 \\
\hline 10 to 15 stools per day & 37.0 \\
\hline More than 15 stools per day & 22.2 \\
\hline Not measurable & 7.4 \\
\hline \multicolumn{2}{|l|}{ Duration of CID, \% } \\
\hline 1 to 3 days & 8.5 \\
\hline 3 to 5 days & 25.4 \\
\hline 5 to 7 days & 20.3 \\
\hline More than 7 days & 45.8 \\
\hline \multicolumn{2}{|l|}{ Conditions associated with CID, \% } \\
\hline Cramping & 39.7 \\
\hline Bloody diarrhea & 9.5 \\
\hline Uncontrolled emesis & 20.6 \\
\hline Fever requiring antibiotic support & 30.2 \\
\hline Stomatitis & 7.9 \\
\hline Neutropenia & 59.7 \\
\hline Febrile neutropenia & 39.7 \\
\hline Other* & 3.2 \\
\hline Combination of the above & 12.7 \\
\hline \multicolumn{2}{|l|}{ Patient impact before hospitalization, \% } \\
\hline Additional clinic visit & 58.7 \\
\hline Emergency department visit & 42.9 \\
\hline Telephone consultation & 1.6 \\
\hline
\end{tabular}

${ }^{*}$ Fatigue, rash, constipation, anorexia and/or dizziness

of diarrhea while in hospital. In addition, subcutaneous octreotide was also used in 13 patients. The median length of stay was eight days with a three-day median duration of intravenous (IV) fluid support (Table 3). A Clostridium difficile test was performed in 47 patients, with a positive test result being 


\section{TABLE 3}

Supportive care administered and hospital length of stay

\begin{tabular}{lc}
\hline Parameter & Distribution $\mathbf{( n = 6 3 )}$ \\
\hline Antidiarrheal medication, \% & \\
Oral antidiarrheals & 58.7 \\
Octreotide alone & 1.6 \\
Octreotide and oral antidiarrheals & 19.0 \\
None & 12.7 \\
Missing data & 7.9 \\
Hospital admission, \% & 100 \\
Median length of stay, days (range) & $8(1$ to 49$)$ \\
Parenteral support, \% & 87.3 \\
Median days IV fluid support (range) & $3(1$ to 24$)$ \\
Clostridium difficile test performed, \% & 74.6 \\
Positive test result, \% & 9.5 \\
Intravenous/oral antibiotics administered & $\%$ \\
\hline
\end{tabular}

${ }^{*}$ Broad spectrum antibiotics for empiric therapy in febrile neutropenia were not included in the analysis

\section{TABLE 4}

Patient outcomes following the chemotherapy-induced diarrhea (CID) event

\begin{tabular}{lc}
\hline Parameter & Distribution $(\mathbf{n}=\mathbf{6 3})^{*}$ \\
\hline Patient death, \% ${ }^{\dagger}$ & 14.3 \\
Evaluable for assessment, \% & 96.8 \\
CID resolution, \% & 88.5 \\
Median time to resolution, days (range) & $12(1$ to 109) \\
Impact on subsequent chemotherapy, \% & \\
Dose reduction only & 9.5 \\
Treatment delay only & 3.2 \\
Both dose and delay reduction & 23.8 \\
Change in chemotherapy regimen & 15.9 \\
Discontinuation of chemotherapy & 34.2 \\
Other $\ddagger$ & 15.0 \\
Median duration of delay, days (range) $(\mathrm{n}=17)$ & $14(7$ to 35$)$ \\
Median dose reduction, \% (range) $(\mathrm{n}=21)$ & $25(10$ to 80$)$ \\
CID recurrence, \%§ & 14.3 \\
\hline
\end{tabular}

*There were seven patients with missing data; there was a total of nine deaths, with three (4.8\%) possibly being related to the diarrhea; $¥$ Treatment continued as before, etc. The estimates do not add up to $100 \%$ because all future chemotherapy was evaluated and events are not mutually exclusive; $\S$ The same or an alternative chemotherapy was continued in 35 patients. Hence, the diarrhea recurred in five of these patients

reported in six cases. Overall, 49 patients received either oral or IV antibiotics for the treatment of their diarrhea. Agents typically used were oral ciprofloxacin and oral/IV metronidazole.

Patient outcomes and the impact that the diarrhea event had on subsequent chemotherapy were then assessed. There were nine patient deaths with uncontrolled diarrhea possibly contributing to three deaths. For the remaining 54 patients that were evaluable for response, the diarrhea was successfully resolved in all cases with a median time to resolution of 12 days (Table 4). Chemotherapy was delayed in 17 patients for a median of 14 days. The dose of chemotherapy was reduced by a median of $25 \%$ in 21 patients. Furthermore, the chemotherapy regimen
TABLE 5

Health care resource utilization associated with the chemotherapy-induced diarrhea event (selection off*)

\begin{tabular}{lc}
\hline Parameter, mean (range) & Distribution (n=63) \\
\hline Hospital days & $9.79(1$ to 49$)$ \\
Emergency department visits & 0.43 (0 to 1$)$ \\
Unplanned oncology clinic visits & 0.60 (0 to 1$)$ \\
Physician visits & 14.3 (2 to 60$)$ \\
Specialist consultations & 0.31 (0 to 1$)$ \\
Estimated nursing support, $h$ & $16.6(1$ to 112$)$ \\
\hline
\end{tabular}

${ }^{*}$ Resources for supportive care drugs, laboratory, microbiological and diag nostic tests not included in the above table because the list was extensive

TABLE 6

Overall cost impact of grade III/IV chemotherapy-induced diarrhea

\begin{tabular}{lc}
\hline Parameter, mean (range) & Distribution $(\mathbf{n = 6 3 )}$ \\
\hline Hospitalization, clinic or emergency visits $^{*}$ & $\$ 6,314$ \\
Supportive care $^{\dagger}$ & $\$ 351$ \\
Medical/nursing staff $^{\ddagger}$ & $\$ 738$ \\
Laboratory/microbiological tests $^{\text {Diagnostic tests }}{ }^{\S}$ & $\$ 411$ \\
Total average cost & $\$ 416$ \\
$(95 \% \mathrm{CI})$ & $\$ 8,230$ \\
\hline
\end{tabular}

*Obtained from the Toronto Sunnybrook Regional Cancer Centre and from the University Health Network, Toronto, Ontario, 2004. ${ }^{+}$Consists of antidiarrheal medication, hydration and antibiotics. $¥ P$ Pysician visits, estimated nursing time for patient care and specialist consultations. ${ }^{\S}$ Consists of chest and other $x$-rays, and computed tomography scans

was changed or discontinued in $15.9 \%$ and $34.2 \%$ of patients, respectively (Table 4 ). In the 35 patients where the same or an alternative chemotherapy regimen was continued, the diarrhea recurred in five cases $(14.3 \%)$.

The final phase of the current study was to estimate the overall cost impact of patients with grade III/IV diarrhea that required a hospital admission. When health care resources such as clinic or emergency department visits, hospital stays, supportive care, hospital staff, laboratory and diagnostic tests for the management of diarrhea were quantified (Table 5), the mean cost was $\$ 8,230$ per patient (Table 6). There were 25 patients admitted to hospital with a diagnosis of both febrile neutropenia and uncontrolled grade III/IV diarrhea. The former toxic event may have contributed to the overall length of stay in these patients. To remove the contaminating effect of febrile neutropenia, a sensitivity analysis was performed where these 25 patients were removed from the analysis. A reanalysis of the data generated a mean cost of $\$ 7,576$ per patient $(95 \% \mathrm{CI}$ $\$ 5,791$ to $\$ 9,361)$. Similarly, if the 37 patients with neutropenia were removed, the mean cost per patient was reduced to $\$ 6,661$ (95\% CI $\$ 4,741$ to $\$ 8,581$ ). When patients with concomitant uncontrolled emesis $(n=13)$ and stomatitis $(n=5)$ were removed from the database, a reanalysis of the cost data revealed a mean cost of $\$ 8,617(95 \% \mathrm{CI} \$ 7,607$ to $\$ 9,627)$ and $\$ 7,981$ (95\% CI $\$ 7,121$ to $\$ 8,841)$ per patient, respectively. The sensitivity analyses of these 'worst case scenarios' suggested that the cost of patients hospitalized for uncontrolled CID remained high, even after the removal of these patients. 


\section{DISCUSSION}

The findings of the current study revealed that uncontrolled diarrhea in patients with colorectal cancer can have a substantial cost impact on health care costs and that the event may contribute to patient mortality. Compared with other toxic events of chemotherapy that often require hospitalization, such as febrile neutropenia, cardiac toxicity and stomatitis, the cost impact of hospitalization secondary to uncontrolled diarrhea is substantially higher. In one Canadian cost of illness analysis in breast cancer patients (8), the cost of febrile neutropenia and cardiac toxicity was $\$ 5,871$ and $\$ 4,626$ (converted into 2004 Canadian dollars using the consumer price index for health care as reported by Statistics Canada) compared with $\$ 8,230$ per patient for diarrhea as estimated in the current study. Similarly, severe mucositis at a cost of $\$ 5,500$ is also substantially lower than the cost of hospitalization secondary to uncontrolled diarrhea (9). Therefore, the ability to either prevent or reduce the intensity of severe diarrhea with agents such as octreotide may be able to avoid hospital admissions and possibly reduce the risk of treatment-related death. To measure the efficacy of octreotide as primary prophylaxis, our group will initiate a randomized trial in rectal patients receiving adjuvant chemotherapy. The results of this trial will provide important information for the updating of guidelines for the prevention and management of diarrhea in cancer patients (10).

There are a number of limitations in the current study that need to be addressed. The current study was retrospective,

\section{REFERENCES}

1. National Cancer Institute of Canada. Canadian Cancer Statistics 2003. Toronto: National Cancer Institute of Canada, 2003.

2. Kornblau S, Benson AB, Catalano R, et al. Management of cancer treatment-related diarrhea. Issues and therapeutic strategies. J Pain Symptom Manage 2000;19:118-29.

3. Grem JL, Shoemaker DD, Petrelli NJ, Douglass HO Jr. Severe life threatening toxicities observed in study using leucovorin with 5-fluorouracil. J Clin Oncol 1987;5:1704-12.

4. Arbuckle RB, Huber SL, Zacker C. The consequences of diarrhea occurring during chemotherapy for colorectal cancer: A retrospective study. Oncologist 2000;5:250-9.

5. Rosenoff S. Octreotide LAR resolves severe chemotherapy-induced diarrhea (CID) and allows continuation of full-dose therapy. Proc Am Soc Clin Oncol 2003;22:A3126. (Abst)

6. Cancer Therapy Evaluation Program, National Cancer Institute. which sometimes created challenges in the accurate determination of the number of stools during the CID event. The total sample size was small $(n=63)$ and selected from only two cancer centres in Canada. This could compromise the generalizability of the results. In addition, only patients with colorectal cancer were considered; therefore, the findings cannot be extended to other disease sites. In some patients, the severe diarrhea was associated with other comorbid events such as febrile neutropenia and uncontrolled emesis. These events could have contributed to the length of hospital stay. A sensitivity analysis partly addressed these limitations and revealed that the cost of severe diarrhea remained substantial. Indirect costs, such as time off work, were not considered which would lead to an underestimation of the overall cost of severe diarrhea.

\section{CONCLUSIONS}

The findings of the current cost of illness analysis revealed that severe diarrhea requiring hospital admission is a costly and potentially fatal complication of chemotherapy in colorectal cancer. The identification of predictive factors for these highrisk patients and the implementation of prophylactic measures could reduce the morbidity and mortality associated with this troublesome event.

FUNDING: The study was funded by an unrestricted research grant from Novartis Pharmaceuticals Canada Inc.

Common toxicity criteria. Version 2.0. Bethesda: National Cancer Institute, National Institutes of Health, US Department of Health and Human Services, 1999.

7. Doyle C, Stockler M, Pintilie M, et al. Resource implications of palliative chemotherapy for ovarian cancer. J Clin Oncol 1997;15:1000-7.

8. Dranitsaris G, Tran TM. Economic analyses of toxicity secondary to anthracycline-based breast cancer chemotherapy. Eur J Cancer 1995;31A:2174-80.

9. Elting LS, Cooksley C, Chambers M, Cantor SB, Manzullo E, Rubenstein EB. The burdens of cancer therapy. Clinical and economic outcomes of chemotherapy-induced mucositis. Cancer 2003;98:1531-9.

10. Wadler S, Benson AB 3rd, Engelking C, et al. Recommended guidelines for the treatment of chemotherapy-induced diarrhea. J Clin Oncol 1998;16:3169-78. 


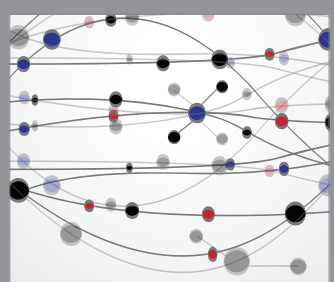

The Scientific World Journal
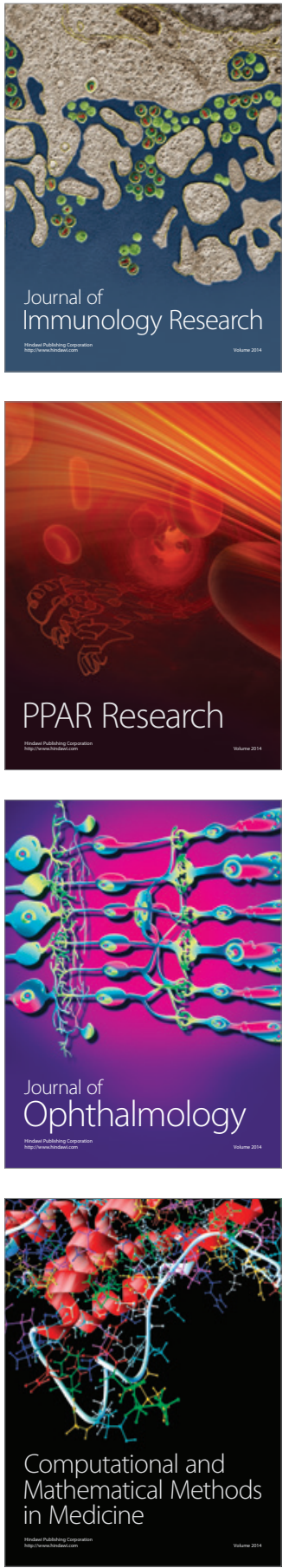

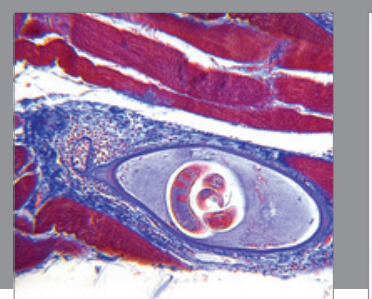

Gastroenterology Research and Practice

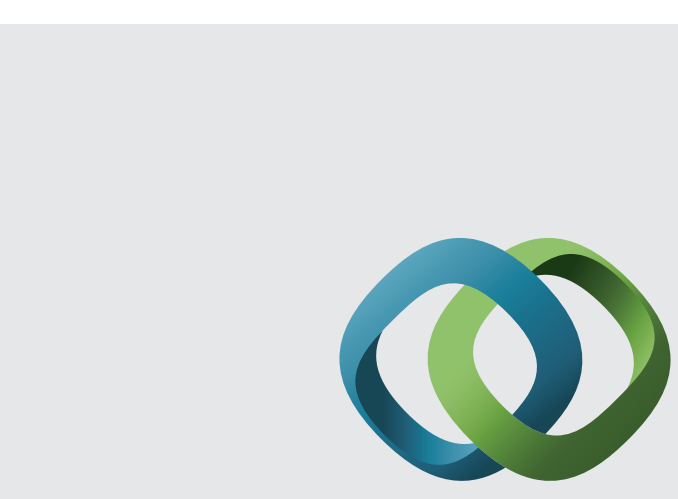

\section{Hindawi}

Submit your manuscripts at

http://www.hindawi.com
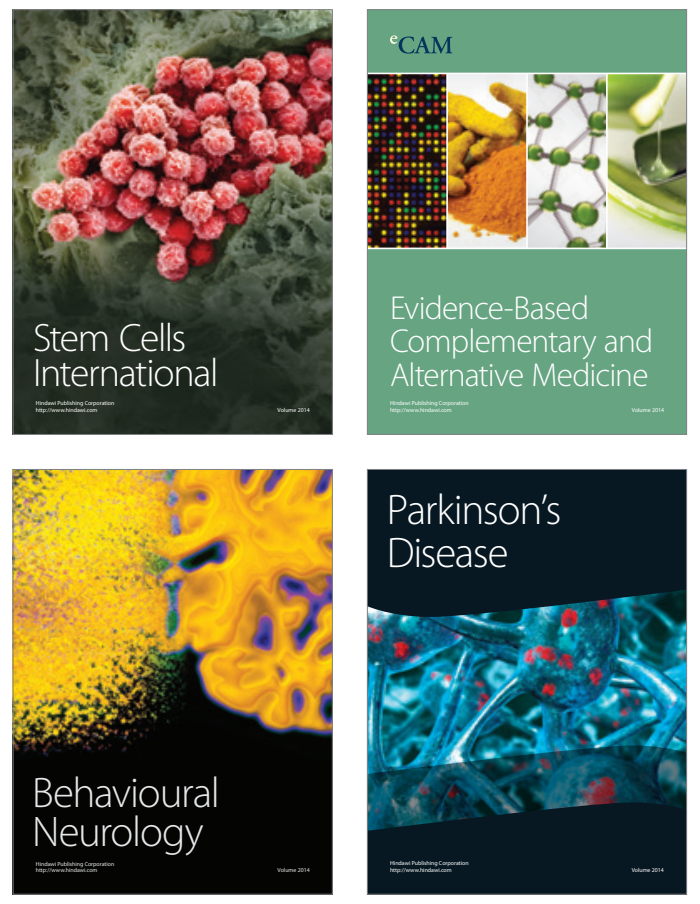
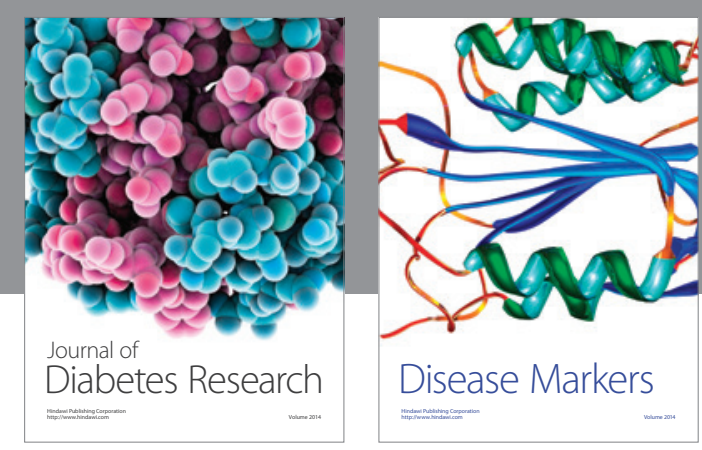

Disease Markers
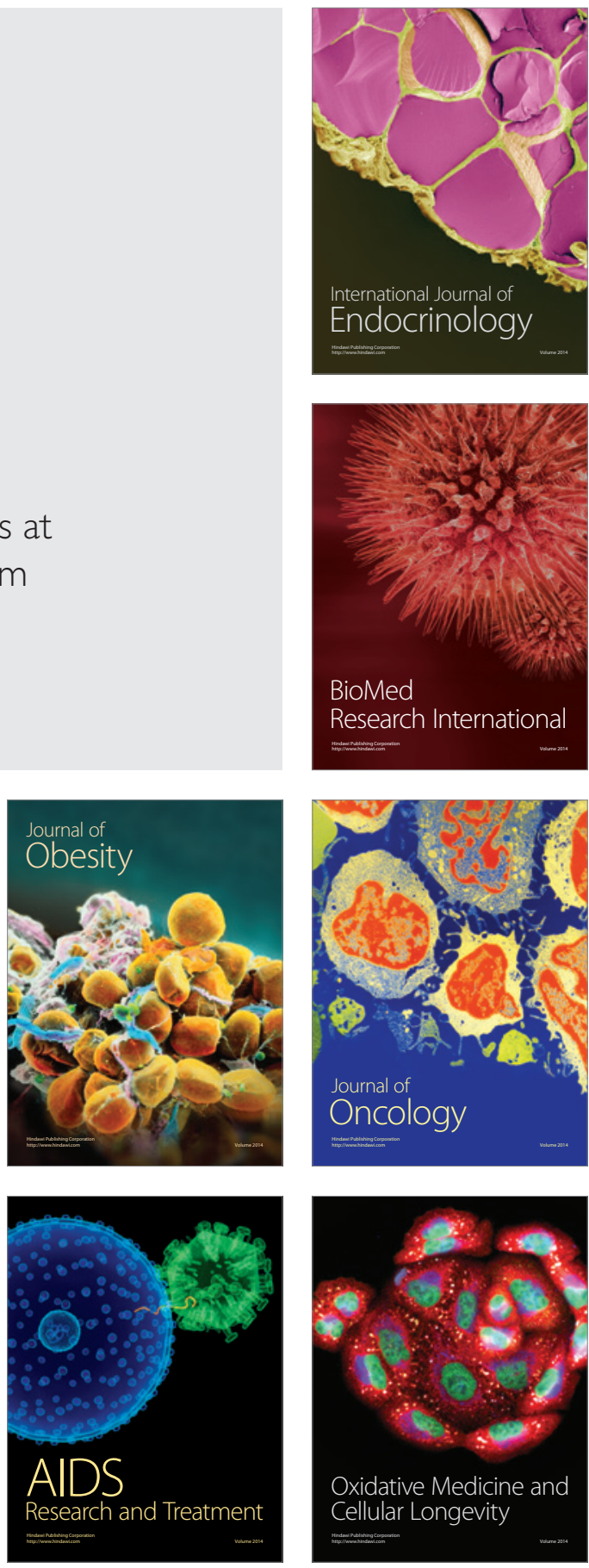